\title{
Areas of Projections of Analytic Sets
}

\author{
H. Alexander (Ann Arbor), B. A. Taylor (Ann Arbor) \\ and J. L. Ullman (Ann Arbor)
}

1.

If $V$ is a pure 1-dimensional analytic subvariety of $\mathbf{C}^{n}$, then the area of $V$ is the sum of the areas, counting multiplicity, of the projections of $V$ onto the $n$ coordinate lines $[5,6]$. If $V$ is a pure 1-dimensional variety in the unit ball in $\mathbf{C}^{n}$ which passes through the origin, then [5] the area of $V$ is at least $\pi$. Together these theorems imply that a 1variety through 0 in the unit ball has the property that the sum of the areas of its projections on the coordinate lines, taken with multiplicity, is bounded below by $\pi$. We shall generalize this result by showing that the conclusion is valid without counting multiplicities. A similar property of higher dimensional varieties will also be obtained. The proof depends upon a one variable result (Theorem 1) which relates the area of the image of a function analytic in the unit disc to its $L^{2}$-norm on the circle. As an application we extend a result of Nishino [4] to the effect that a family of varieties is locally finite if each family of coordinate slices is such. This in turn will be applied to complete a theorem of Rothstein [7] on characterizing analytic sets in $\mathbf{C}^{n}$ as those whose coordinate slices are analytic sets.

2.

We begin with the basic one variable result. Here $D$ will denote the open unit disc $\{z \in \mathrm{C}:|z|<1\}$ and $N$ the usual Nevanlinna space of analytic functions of bounded characteristic on $D[2, \mathrm{p} .16]$. For $f \in N$, $f^{*}$ will denote the boundary function

defined for almost all $\theta$.

$$
f^{*}\left(e^{i \theta}\right)=\lim _{r \rightarrow 1^{-}} f\left(r e^{i \theta}\right)
$$

Theorem 1. Let $f \in N$ and $f(0)=0$. Then

$$
\frac{1}{2} \int_{0}^{2 \pi}\left|f^{*}\left(e^{i \theta}\right)\right|^{2} d \theta \leqq \operatorname{arca}(f(D))
$$

This theorem follows by integrating, with respect to $t$, from 0 to $+\infty$ in the following inequality [3, pp. 421-422].

22 Inventiones math., Vol. 16 
Lemma. Let $f \in N, f(0)=0$. Then for $t>0$,

$$
t \ell\left\{e^{i \theta}:\left|f^{*}\left(e^{i \theta}\right)\right|>t\right\} \leqq \ell\left(f(D) \cap \Gamma_{t}\right)
$$

where $\Gamma_{t}=\{w:|w|=t\}$ and $\ell$ is arc length measure.

Proof. Fix $r, 0<r<1$, and $t>0$. Put $E_{t}=\left\{e^{i \theta}:\left|f_{r}\left(e^{i \theta}\right)\right|>t\right\}$ where $f_{r}(z)=f(r z)$. Now let $u(z)$ be the bounded harmonic function in $D$ with boundary values equal to 0 if $e^{i \theta} \notin E_{t}$ and -1 if $e^{i \theta} \in E_{t}$. That is,

$$
u(z)=\frac{-1}{2 \pi} \int_{E_{t}} \operatorname{Re}\left(\frac{e^{i \theta}+z}{e^{i \theta}-z}\right) d \theta .
$$

Note that $u(0)=\frac{-1}{2 \pi} \ell\left(E_{t}\right)$, and that

$$
\left|f_{r}\left(e^{i \theta}\right)\right|<t \Rightarrow \lim _{z \rightarrow e^{i \theta}} u(z)=0 .
$$

Now, define $\varphi(w)$ for $|w| \leqq t$ by

$$
\varphi(w)= \begin{cases}\min \left\{u(z): f_{r}(z)=w, z \in D\right\} & \text { if } w \in f_{r}(D) \\ 0 & \text { if } w \notin f_{r}(D) .\end{cases}
$$

We claim that $\varphi$ is superharmonic in $|w|<t$.

We will assume that $E_{t} \neq \emptyset$, since otherwise (2.5) below is trivial. Therefore, since $f_{r}$ is analytic on $|z|=1$, the set

$$
F_{t}=\left\{e^{i \theta}:\left|f_{r}\left(e^{i \theta}\right)\right|=t\right\}
$$

is finite. Now $\varphi$ is continuous on $|w| \leqq t$ except possibly for the finite set $f_{r}\left(F_{t}\right) \subset\{w:|w|=t\}$. Off the finite set $X$ of critical values of $f_{r}$ in $|w| \leqq t$, (2.1) implies that $\varphi$ is locally the minimum of a finite number of harmonic functions, hence superharmonic off $X$. Since $\varphi$ is continuous in $|w|<t$ and $X$ is discrete, it follows that $\varphi$ is superharmonic in $|w|<t$.

In particular, we have

$$
\varphi(0) \geqq \frac{1}{2 \pi} \int_{-\pi}^{\pi} \varphi\left(t e^{i \theta}\right) d \theta
$$

Now, $\varphi\left(t e^{i \theta}\right)=0$ unless $t e^{i \theta} \in \overline{f_{r}(D)}$ in which case $\varphi\left(t e^{i \theta}\right) \geqq-1$ because $u(z) \geqq-1$ for $z \in D$. Thus, we get from (2.2),

$$
\varphi(0) \geqq \frac{-1}{2 \pi} \frac{\ell\left(\overline{f_{r}(D)} \cap \Gamma_{t}\right)}{t} .
$$

On the other hand, since $f_{r}(0)=0$, we have

$$
\varphi(0) \leqq u(0)=-\frac{1}{2 \pi} \ell\left(E_{t}\right) .
$$


From (2.3) and (2.4) it follows that

But, $\overline{f_{r}(D)} \subset f(D)$, so

$$
t \ell\left(E_{t}\right) \leqq \ell\left(\overline{f_{r}(D)} \cap \Gamma_{t}\right)
$$

$$
t \ell\left\{e^{i \theta}:\left|f_{r}\left(e^{i \theta}\right)\right|>t\right\} \leqq \ell\left(f(D) \cap \Gamma_{t}\right) .
$$

Letting $r \rightarrow 1^{-}$gives the desired result because $f_{r}\left(e^{i \theta}\right) \rightarrow f^{*}\left(e^{i \theta}\right)$ a.e., and therefore also in measure. Q.E.D.

Remark. The superharmonic function $\varphi$ of the proof was essentially introduced by Nishino [4, p. 259] for a related problem.

In the next theorem, $B$ denotes the unit ball in $\mathbf{C}^{n}$,

$$
\left\{z=\left(z_{1}, \ldots, z_{n}\right): \sum_{1}^{n}\left|z_{i}\right|^{2}<1\right\} \text {. }
$$

Theorem 2. Let $V$ be a pure 1-dimensional analytic subvariety of $B$ containing the origin. Then the sum of the areas of the projections of $V$ on the $n$ coordinate lines is at least $\pi$.

Proof. We may assume that $V$ is irreducible. Let $V^{*}$ be the normalization of $V, \tau: V^{*} \rightarrow V$ the associated projection. Since $V^{*}$ carries nonconstant bounded analytic functions, the uniformization theorem for Riemann surfaces implies that the universal covering surface of $V^{*}$ is $D$, the unit disc. Let $\Psi: D \rightarrow V^{*}$ be the universal covering map, chosen so that $(\tau \circ \Psi)(0)=0$. Now $\tau \circ \Psi$ is given by $n$ analytic functions $f_{1}, \ldots, f_{n}$ on $D$ with $f_{j}(0)=0$ and $\sum_{i}^{n}\left|f_{j}(z)\right|^{2}<1$.

The $f_{i}$ are bounded and have boundary functions $f_{i}^{*}$. We claim that: a.e. $d \theta$

$$
\sum_{1}^{n}\left|f_{j}^{*}\left(e^{i \theta}\right)\right|^{2}=1
$$

In fact, let $S=\left\{e^{i \theta}\right.$ : each $f_{j}, 1 \leqq j \leqq n$, has a radial limit at $\left.e^{i \theta}\right\}$. The complement of $S$ is a null set as each $f_{j}$ has a radial limit a.e. $d \theta$. We will show that (2.6) holds for $e^{i \theta} \in S$. Suppose not, then there is $e^{i \theta_{0}} \in S$ such that $\sum\left|f_{i}^{*}\left(e^{i \theta_{0}}\right)\right|^{2}<1$. Then $\alpha=\left(f_{1}^{*}\left(e^{i \theta_{0}}\right), \ldots, f_{n}^{*}\left(e^{i \theta_{0}}\right)\right) \in B$ and for $0 \leqq t \leqq 1$, $t \rightarrow(\tau \circ \Psi)\left(t e^{i \theta_{0}}\right)$ is a path in $V$ from 0 to $\alpha$. It follows that for $0 \leqq t \leqq 1$, $t \mapsto \Psi\left(t e^{i \theta_{0}}\right)$ is a path in $V^{*}$ from $\Psi(0)$ to some point $\alpha^{*}$ with $\tau\left(\alpha^{*}\right)=\alpha$. This path, however, does not lift to a (compact) path in $D$, with initial point 0 and this contradicts the fact that $\Psi$ is a covering projection. Thus (2.6) holds on $S$.

Now apply Theorem 1 to $f_{j}$ :

$$
\frac{1}{2} \int_{0}^{2 \pi}\left|f_{j}^{*}\left(e^{i \theta}\right)\right|^{2} d \theta \leqq \operatorname{area}\left(f_{j}(D)\right)
$$


Adding for $1 \leqq j \leqq n$ and using (2.6) we get

$$
\pi \leqq \sum_{j=1}^{n} \operatorname{area}\left(f_{j}(D)\right)
$$

But $f_{j}(D)$ is the projection of $V$ to the $j$-th coordinate line. Q.E.D.

Remark. An alternate proof of Theorem 2 in the context of polynomial convexity and based upon the elements of Banach algebras will appear in [1].

Next we want to generalize Theorem 2 to higher dimensional varieties. If $\alpha$ is a $k$-tuple of integers $\left(i_{1}, i_{2}, \ldots, i_{k}\right)$ with $1 \leqq i_{j} \leqq n$, we denote the length, $k$, of $\alpha$ by $|\alpha|$ and by $\pi_{\alpha}$ the projection $\mathbf{C}^{n} \rightarrow \mathbf{C}^{k}$ : $\pi_{\alpha}\left(z_{1}, \ldots, z_{n}\right)=\left(z_{i_{1}}, z_{i_{2}}, \ldots, z_{i_{k}}\right)$. It is known that if $V$ is a pure $k$-dimensional analytic subvariety through 0 in the unit ball in $\mathbf{C}^{n}$, then the sum of the $2 k$-dimensional measures of the sets $\pi_{x}(V)$ counted with multiplicity and taken over increasing $\alpha$ 's is bounded below by $\pi^{k} / k !=$ the $2 k$-volume of the unit ball in $\mathbf{C}^{k}$. In fact, this sum is just the $2 k$-volume of $V$ [5]. We would like to show that the result remains valid without counting multiplicity. We have

Theorem 3. There are positive constants $C_{k}$ such that if $V$ is a pure $k$-dimensional subvariety of the unit ball in $\mathbf{C}^{n}$ passing through 0 then

$$
\sum_{|\alpha|=k}^{\prime} \lambda_{2 k}\left(\pi_{\alpha}(V)\right) \geqq C_{k}
$$

where the prime denote summation over increasing $\alpha$ 's and $\lambda_{2 k}$ is Lebesque measure in $\mathbf{C}^{k}$.

Remark. The constants $C_{k}$ are independent of $n$. A natural conjecture is that $\pi^{k} / k$ ! can be chosen for $C_{k}$ but we are unable to prove this unless $k=1$.

Proof. We proceed by induction on $k$, assuming the result for $j<k$ (for $k=1$ this is just Theorem 2). Let $B(p, r)$ be the Euclidean ball about $p$ of radius $r$ and let $W=V \cap B\left(0, \frac{1}{2}\right)$. Since $W$ contains a 1-dimensional variety through 0 , we apply the 1-dimensional result in the ball of radius $\frac{1}{2}$ (necessitating a scale change) and conclude:

$$
\sum_{j=1}^{n} \lambda_{2}\left(\pi_{j}(W)\right) \geqq\left(\frac{1}{2}\right)^{2} \pi
$$

Consider $\pi_{n}(W)$. If $\zeta \in \pi_{n}(W)$, there is $\zeta^{\prime} \in \mathbf{C}^{n-1}$ such that $q=\left(\zeta^{\prime}, \zeta\right) \in V$ and $\|q\|<\frac{1}{2}$. Let $H_{\zeta}=\left\{z \in \mathbf{C}^{n}: \pi_{n}(z)=\zeta\right\}$ and consider the $(k-1)$-variety $H_{\zeta} \cap V$ (we may assume that $V$ is irreducible so that $\operatorname{dim} H_{\zeta} \cap V<\operatorname{dim} V$ ). Now $q \in H_{\zeta} \cap V$ and we may apply the $(k-1)$-dimensional result to 
$H_{\zeta} \cap V \cap B\left(q, \frac{1}{2}\right)$ in $B\left(q, \frac{1}{2}\right)$ and obtain:

$$
\sum_{\substack{|\beta|-k-1 \\ n \notin \beta}}^{\prime} \lambda_{2 k-2}\left(\pi_{\beta}\left(H_{\zeta} \cap V\right)\right) \geqq\left(\frac{1}{2}\right)^{2 k-2} C_{k-1} .
$$

This sum is over $\beta$ with $n \notin \beta$ since $\pi_{n} \equiv \zeta$ on $H_{\zeta} \cap V$. Integrating over $\pi_{n}(W)$ we get

$$
\sum_{\substack{|\beta|=k-1 \\ n \notin \beta}}^{\prime} \int_{\pi_{n}(W)} \lambda_{2 k-2}\left(\pi_{\beta}\left(H_{\zeta} \cap V\right)\right) d \lambda_{2}(\zeta) \geqq\left(\frac{1}{2}\right)^{2 k-2} C_{k-1} \lambda_{2}\left(\pi_{n}(W)\right) .
$$

Applying Fubini's theorem to the integrals on the left yields

$$
\sum_{\substack{|\beta|=k-1 \\ n \notin \beta}}^{\prime} \lambda_{2 k}\left(\pi_{(\beta, n)}(V)\right) \geqq\left(\frac{1}{2}\right)^{2 k-2} C_{k-1} \lambda_{2}\left(\pi_{n}(W)\right) .
$$

Now the replacing in (2.8) of $n$ by $j$, with $1 \leqq j \leqq n$, leads to $n$ inequalities of the same type. Adding these and recalling (2.7) we get

$$
\sum_{j=1}^{n} \sum_{\substack{|\beta|=k-1 \\ j \notin \beta}}^{\prime} \lambda_{2 k}\left(\pi_{(\beta, j)}(V)\right) \geqq\left(\frac{1}{2}\right)^{2 k} \pi C_{k-1} .
$$

For a fixed $\alpha$ with $|\alpha|=k$ there are at most $k(\beta, j)$ 's such that $(\beta, j)=\alpha$ as sets of $k$ integers. Thus each $2 k$-volume term in the left hand side of (2.9) is repeated at most $k$ times. It follows that we may choose $C_{k}$ to be $\pi C_{k-1} / k 2^{2 k}$. Q.E.D.

3.

As an application we have

Theorem 4. Let $\mathscr{V}$ be a family of pure k-dimensional analytic subvarieties in an open subset $\Omega$ of $\mathbf{C}^{n}$. Suppose that for every coordinate $(n-k)$-plane $H\left(=a n\right.$ affine linear subspace of $\mathbf{C}^{n}$ obtained by fixing $k$ of the coordinates), the family $\mathscr{V}_{H}=\{V \cap H: V \in \mathscr{V}\}$ is locally finite. Then $\mathscr{V}$ is locally finite.

Remark. This extends a result of Nishino [4] who treated the case of varieties of codimension 1 .

Proof. We argue by contradiction. If $\mathscr{V}$ is not locally finite then there is $p \in \Omega$ and distinct $\left\{V_{j}\right\}_{1}^{\infty} \subseteq \mathscr{V}$ with $p_{j} \in V_{j}$ such that $p_{j} \rightarrow p$. Choose $r>0$ such that $\overline{B(p, r)} \subseteq \Omega$. Let $W_{j}=V_{j} \cap B(p, r)$, and let $\alpha$ be a $k$-tuple. If $\zeta \in \mathbf{C}^{k}$, then $\pi_{\alpha}^{-1}(\zeta)$ is a coordinate $n-k$ plane and so $W_{j} \cap \pi_{\alpha}^{-1}(\zeta)$ is empty for large $j$ by hypothesis. That is, the sets $\pi_{\alpha}\left(W_{j}\right)$ eventually omit every point in $B\left(\pi_{\alpha}(p), r\right)$. It follows that $\lambda_{2 k}\left(\pi_{\alpha}\left(W_{j}\right)\right) \rightarrow 0$ for every $\alpha$. But by Theorem 3 
a finite sum of such terms is bounded below by a number approaching $r^{2 k} C_{k}$ (since $p_{j} \rightarrow p$ ). This is a contradiction. Q.E.D.

Theorem 5. Let $A$ be a subset of an open set $\Omega \subseteq \mathbf{C}^{n}$. Let $r \geqq 1$ be an integer. Suppose that the intersection of $A$ with every coordinate hyperplane (i.e. every coordinate $(n-1)$-plane) is a pure r-dimensional subvariety of $\Omega$. Then $A$ is a subvariety of $\Omega$ (of dimension $r+1$ ).

Remark. Rothstein proved this proposition under the further assumption that $A$ is a (relatively) closed subset of $\Omega$ in [7] and asked there if this restriction was really needed. What we shall prove here is simply that the hypotheses of the theorem force $A$ to be a relatively closed subset of $\Omega$.

Proof. In order to show that $A$ is a relatively closed subset of $\Omega$ it is enough to show that if $0 \in \bar{A} \cap \Omega$ then $0 \in A$. We argue by contradiction and suppose otherwise; i.e. that $0 \in \bar{A} \cap \Omega$ but $0 \notin A$. Then if $H$ is the coordinate hyperplane $\left\{z: \pi_{n}(z)=0\right\}, A \cap H$ is a subvariety of $\Omega$ and hence relatively closed. Consequently, as $0 \notin A$ there is $\delta>0$ such that

$$
\left\{z:\left|z_{1}\right| \leqq \delta,\left|z_{2}\right| \leqq \delta, \ldots,\left|z_{n-1}\right| \leqq \delta, z_{n}=0\right\} \cap A=\varnothing
$$

and by choosing $\delta$ small enough we may assume that

$$
\left\{z:\left|z_{j}\right| \leqq \delta, 1 \leqq j \leqq n\right\} \subseteq \Omega .
$$

As $0 \in \bar{A}$ there are $\left(c_{j}, c_{j}^{\prime}\right) \in\left(\mathbf{C}^{n-1} \times \mathbf{C}\right) \cap A$ such that $c_{j}^{\prime} \rightarrow 0, c_{j} \rightarrow 0$ and $c_{j}=\left(c_{1}^{j}, \ldots, c_{n-1}^{j}\right) \in \mathbf{C}^{n-1}$ with each $\left|c_{s}^{j}\right|<\delta$ and $\left|c_{j}^{\prime}\right|<\delta$.

Let $\Omega_{1}=\left\{z \in \mathbf{C}^{n-1}:\left|z_{s}\right|<\delta, 1 \leqq s \leqq n-1\right\}$, let $\tilde{\pi}: \mathbf{C}^{n} \rightarrow \mathbf{C}^{n-1}$ be the projection $\tilde{\pi}\left(z_{1}, \ldots, z_{n}\right)=\left(z_{1}, \ldots, z_{n-1}\right)$ and let

$$
W_{j}=\tilde{\pi}\left(A \cap\left\{z:\left|z_{1}\right|<\delta, \ldots,\left|z_{n-1}\right|<\delta, z_{n}=c_{j}^{\prime}\right\}\right) .
$$

Then $W_{j}$ is a $r$-dimensional subvariety of $\Omega_{1}$ and $\left\{W_{j}\right\}$ is not a locally finite family as $W_{j} \ni c_{j} \rightarrow 0$. Hence by Theorem 4 , the intersections of the $W_{j}^{\prime}$ s with some coordinate $(n-1)-r$ plane is not locally finite and consequently there is a coordinate $n-2$ plane $K$ in $\mathbf{C}^{n-1}$ such that the sets $K \cap W_{j}$ are not eventually empty. Passing to a subsequence we may assume that $K \cap W_{j}$ is never empty. Without loss of generality, we may assume that $K=\left\{z \in \mathbf{C}^{n-1}: z_{1}=\lambda\right\}$ where $|\lambda|<\delta$. This means that there are points $d_{j}=\left(d_{1}^{j}, \ldots, d_{n-2}^{j}\right) \in \mathbf{C}^{n-2}$ such that each $\left|d_{s}^{j}\right|<\delta$ and $\left(\lambda, d_{j}\right) \in W_{j}$. Hence $\left(\lambda, d_{j}, c_{j}^{\prime}\right) \in A$. By passing to a subsequence, we may assume that $d_{s}^{j} \rightarrow d^{j}$ with $\left|d^{j}\right| \leqq \delta$. Put $d=\left(d^{1}, d^{2}, \ldots, d^{n-2}\right)$. Now $A \cap\left\{z: z_{1}=\lambda\right\}$ is a relatively closed subset of $\Omega$ and contains the points $\left(\lambda, d_{j}, c_{j}^{\prime}\right)$ which converge to $(\lambda, d, 0) \in A$. But this contradicts (3.1) and we conclude that $A$ is relatively closed in $\Omega$. Q. E. D. 


\section{References}

1. Alexander, H.: Projections of polynomial hulls (to appear).

2. Duren, P.: Theory of $H^{p}$ spaces. New York: Academic Press 1970.

3. Hewitt, E., Stromberg, K.: Real and abstract analysis. Berlin-Heidelberg-New York: Springer 1965.

4. Nishino, T.: Sur une propriété des familles de fonctions analytiques de deux variables complexes. J. Math. Kyoto Univ. 4-2, 255-282 (1965).

5. Stolzenberg, G.: Volumes, limits, and extensions of analytic varieties. Lecture Notes in mathematics 19. Berlin-Heidelberg-New York: Springer 1966.

6. Rham, G. de: On the area of complex manifolds, global analysis (volume dedicated to K. Kodaira), pp. 141-148. Tokyo Univ. Press and Princeton Univ. Press, 1969.

7. Rothstein, W.: Zur Theorie der analytischen Mengen. Math. Ann. 174, 8-32 (1967).

H. Alexander

B. A. Taylor

J.L. Ullman

The University of Michigan

Department of Mathematics

347 West Engineering Building

Ann Arbor, Mich. 48104

USA

(Received February 12.1972) 